\title{
Sub- and supralexical information in early phases of lexical access
}

\author{
Juhani Järvikivi ${ }^{1,2}$ * and Pirita Pyykkönen ${ }^{3}$ \\ ${ }^{1}$ Department of Modern Languages, Norwegian University of Science and Technology, Trondheim, Norway \\ 2 Institute of Behavioral Sciences, University of Helsinki, Helsinki, Finland \\ ${ }^{3}$ Department of Computational Linguistics, Saarland University, Saarbrücken, Germany
}

\section{Edited by:}

Charles Jr. Clifton, University of

Massachusetts Amherst, USA

Reviewed by:

Harald Baayen, University of Alberta,

Canada

Alexander Pollatsek, University of

Massachussetts Amherst, USA

${ }^{*}$ Correspondence:

Juhani Järvikivi, Language Acquisition and Language Processing Lab,

Department of Modern Languages, Norwegian University of Science and Technology, 7491 Trondheim, Norway. e-mail: jjarvikivi@gmail.com
The present study investigated sub- and supralexical effects in morphological processing for inflected and pseudo complex words and pseudo words in lexical decision with masked and cross-modal priming. The results showed that the early stage of morphological processing is not only sensitive to whether the orthographic string can be segmented into an existing stem and affix, but also whether the full form is an existing word the meaning of which differs from the meaning of the segmented stem. It is thus likely that from early on morphological processing is probably not governed by morpho-orthographic processes alone, but is most likely sensitive to top-down information, perhaps originating from supralexical semantic connections between the words morphological family members. In addition, whereas semantic interpretability has a clear advantage later in processing, this stage seems to be sensitive to bottom-up form information as well. In a detailed theoretical discussion we show how these findings, along with earlier findings, are explained by a model that assumes that morphological information is represented at two interactive levels, corresponding to sublexical form (orthographic) and supralexical (semantic) information mediated by a lexical level. This allows supralexical (semantic) effects to feed top-down, predicting differences between regular inflected and pseudo complex words at the lexical level, affecting the early phases of processing for these words.

Keywords: morphology, semantics, masked priming, cross-modal priming, lexical access, morphological family size, pseudo complex words, inflection

\section{INTRODUCTION}

Such central and fundamental question as how and when the language processor accesses the meaning of complex words has turned out to be a challenging task to resolve. For example, when people encounter the word lions, the word may be first segmented into its parts reflecting the morphological structure (lion - stem; $s$-affix), and these features would again get integrated later in processing. Even though many studies indicate that the recognition of morphologically complex words takes place in stages, including processes such as sublexical segmentation of stems, affixes, and roots, there is yet no consensus as to what kind of information the processor receives at different stages of lexical access.

One line of research assumes that no information beyond the pure form of the word is available at the earliest stage of morphological segmentation (Longtin et al., 2003; Rastle et al., 2004; Longtin and Meunier, 2005; Meunier and Longtin, 2007; MarslenWilson et al., 2008; Solomyak and Marantz, 2009). In other words, these studies suggest that the process at this early sublexical stage is sensitive purely to formal morpho-orthographic features of the word, and, thus, blind to information such as (morpho)semantics. This kind of information, which we will call supralexical, would be important only later at the integration phase when different features are combined together (e.g., Meunier and Longtin, 2007). However, recent studies have challenged this view by proposing that also supralexical information originating from above the form level might be used already during the early morphological segmentation phase (Perea and Gotor, 1997; Bodner and Masson, 2003; Bowers et al., 2005; Feldman and Basnight-Brown, 2008; Feldman et al., 2009; Järvikivi et al., 2009).

In what follows, we will first present detailed evidence for the sub- and supralexical effects in morphological processing. We will then discuss frameworks of morphological processing we dub morphology-first models, i.e., models that allow for morphologically motivated information to affect lexical processing at the earliest stages of the processing of complex words. We will then outline the predictions driven from the current models and present a study testing some of the remaining open hypotheses.

\section{(LACK OF) EVIDENCE FOR SUPRALEXICAL EFFECTS AT THE EARLY PROCESSING STAGES}

Recent studies have argued that the early segmentation phase operates only on the formal properties of the input string, and is thus blind to the meaning of the string or indeed to any feedback from top-down. Strikingly, recent studies that have used a form of lexical priming in which a forward masked prime word is presented for a very short time, approximately $50 \mathrm{~ms}$, prior to the target word, have observed significant priming for simplex prime words that are only apparently complex (hence, pseudo complex), like corner, but bear no lexical relation to the respective target word, corn; whereas no comparable facilitation has usually been 
observed for pairs like turnip-turn in which the ending -ip is not an existing affix (Longtin et al., 2003; Rastle et al., 2004). Further evidence shows that this generalizes to "complex" pseudo words consisting of an illegal combination of a stem and an affix, e.g., rapidifier "quickify" (Longtin and Meunier, 2005; for French); as well as to real inflected words that share a bound stem with the prime lexeme, but whose full form-meaning is not related to it, like Finnish aito-na "genuine-as" - aita "fence" (Järvikivi et al., 2009). These results suggest that significant facilitation occurs as long as the prime is potentially segmentable into an existing stem and an existing affix, irrespective of whether the prime shares any meaning with the target or not. These findings have been taken as evidence against morphology-second accounts that assume that morphology has an effect only after full word form is resolved, and therefore, assume no priming in such cases irrespective of whether they are segmentable (corner) or not (turnip; e.g., Giraudo and Grainger, 2000). Instead, the findings are interpreted supporting morphology-first accounts that assume that morphological decomposition is an automatic process, which occurs very early, is triggered by formal properties, and ignores information from higher levels, such as semantics (e.g., Taft and Kougious, 2004; Marslen-Wilson et al., 2008; Taft and Nguyen-Hoan, 2010).

However, as noted by Järvikivi et al. (2009), in several studies, the priming effects for the pseudo complex words tend to be systematically smaller than those for transparent derived words. Indeed, the review by Feldman et al. (2009) confirmed this: in 12 of the 16 reviewed experiments, even if statistically indistinguishable, facilitation was numerically smaller for (opaque) pseudo complex words than for (transparent) derived primes (see also Taft and Nguyen-Hoan, 2010; cf. Rastle and Davis, 2008). One explanation for this is that supralexical information affects processing already at this early stage. In many studies this information is argued to be semantic in nature. For example, Diependaele et al. (2005) argued that the early morphological processing phase involves two parallel processes, one based on morpho-orthographic and another on morpho-semantic correspondence. They used both masked intramodal visual and cross-modal priming: a visual prime word was shown briefly $(67 \mathrm{~ms})$ masked between a forward $(500 \mathrm{~ms})$ mask consisting of a line of hashmarks and a backward mask (13 ms) consisting of a pseudorandom sequence of upper case consonants and followed by a visual target (intramodal) or an auditory target (cross-modal). The results showed significant priming for pseudo complex, corner type, words only in the intramodal task suggesting that the effect could be modality-specific and occur in a very early short lived time window. If they were modalityfree, the effects should have occurred also in the cross-modal task. In addition, Feldman and Basnight-Brown (2008) found that the composition of filler items alter masked priming effects in the following way: Morphological facilitation was found irrespective of whether the fillers were mixed (prime and target orthographically, morphologically, and semantically related), identity (prime and target identical), or semantically related (bug-ant). However, facilitation was stronger with the latter two. Interestingly, priming occurred only with semantically related and identity fillers. Recently, Feldman et al. (2009), compared also semantically transparent, coolant-cool, and opaque pseudo derived pairs, rampant-ramp, matching the affixes in the two conditions. They found greater facilitation for derived than for pseudo complex words. These results suggest that semantic effects may be obtainable in masked priming. Notably, this is also the conclusion of the meta-analysis of Van den Bussche et al. (2009).

Even though these studies still assume that morphoorthographic information is used at the early stage, they do suggest that this segmentation phase may not be as blind to top-down information as suggested earlier. The morphology-first accounts that explain these priming effects as originating from the formlevel representations alone - because according to this view there should be no difference between opaque and transparent primes would find it challenging to incorporate and explain the above findings. In other words, the blind decomposition approach entails that segmentation is based solely on the formal properties, and any morpho-semantic incompatibility is checked only at a later integration stage where strict semantic interpretability is the decisive criterion for whether the lexical representation of the target is activated or not (Meunier and Longtin, 2007). Thus, these assumptions expect the early and late stages to be qualitatively different from each other.

However, if supralexical information indeed affects morphological processing early on, the question arises as to when during processing this information affects lexical access. This is an important question, because earlier studies have shown that it is notoriously difficult to get "pure" lexical-semantic facilitation, i.e., priming based on existing lexical-semantic connections between the prime and the target, like semantic category similarity tigerlion, in masked priming (see Van den Bussche et al., 2009 for an overview). Järvikivi et al. (2009) suggested that because these studies contrast pseudo complex words with derived words, it is possible that the results highlight the similarity between the two word types. In other words, whereas it is generally agreed that derivation always changes the meaning of its base word (Jackendoff, 1975; Aronoff, 1976), this is generally not the case with inflection. Therefore derivation produces new words, and derived forms - like pseudo complex words - have separate lexical (conceptual or lemma) representations (e.g., Taft, 1991; Crepaldi et al., 2010). Strictly speaking, then, a word like bomber would be semantically distinct from its base bomb (in the sense of these words having different meanings and separate lemma representations/lexical entries), just as a word like corner would be from its apparent (pseudo) base corn, despite corner being semantically also clearly unrelated to corn. Therefore, in order to assess the issue of semantic transparency, comparing pseudo complex words with inflected rather than derived words can help to better understand morphological segmentation in morphology-first models.

\section{MORPHOLOGY-FIRST MODELS}

The blind bottom-up decomposition approach predicts no difference between pseudo complex, derived, and inflected words. In fact, any such difference would be troublesome for these approaches, and would suggest the involvement of higher-level linguistic information from early on. In turn, strict morphologysecond approaches (e.g., Giraudo and Grainger, 2000) that assume that any morphological effects would take place only at the supralexical stage after whole word access has been completed would predict that only the inflected transparent words produce 
facilitation, and could therefore not explain why corner would prime corn but turnip would not prime turn (see Järvikivi et al., 2009 for further discussion).

As the above blind morphology-first account and the strict supralexical morphology-second account seem to fail in explaining the current experimental findings, there is a need for a more fine-grained account. One possibility entertained in the previous literature is that priming effects arise as a direct consequence of the strength of form-meaning mappings, morphology being an intermediate emergent property of these mappings (e.g., Plaut and Gonnerman, 2000). This account would potentially account for the graded nature of semantic transparency (e.g., Feldman and Basnight-Brown, 2008); however, it is currently not clear how the observed difference between corner and turnip type of words would arise in this type of model (e.g., Longtin et al., 2003; Rastle et al., 2004; cf. Feldman et al., 2009).

One possibility is to explain these effects as arising at different levels of processing. Indeed, many models of morphological processing assume that morphological information is represented at two levels (Günther, 1988; Kelliher and Henderson, 1990; Schreuder and Baayen, 1995; Baayen et al., 1997; Allen and Badecker, 1999; Järvikivi and Niemi, 2002, 2003). According to the two-level view, the first level representation would code purely formal, e.g., orthographic, properties of words and act as an index to the next level of representation (Aronoff, 1994; Järvikivi and Niemi, 2002). This next, lemma, level would be a modality independent stage coding the words lexical and syntactic properties and acting as an intermediate level between word forms and the semantic system (Levelt et al., 1999; Järvikivi and Niemi, 2003; Crepaldi et al., 2010); corresponding to the access and concept nodes in Schreuder and Baayen (1995) and reformulated as form and lemma by Baayen et al. (1997). Even though this is by no means a new idea in morphological processing (e.g., Günther, 1988; Kelliher and Henderson, 1990; Schreuder and Baayen, 1995; Allen and Badecker, 1999; Järvikivi and Niemi, 2002), it was only recently that top-down feedback between these two stages was argued to be a component of morphological processing (Järvikivi et al., 2006; Crepaldi et al., 2010). Järvikivi et al. (2006) showed that (morpho)phonological transparency affected lexical decision to derived words in Finnish. More precisely, they showed that words formed with transparent derivational affixes, like -sto in kirja-sto "lit. collection of books > library," that retain the form of the affix throughout the inflectional paradigm are more likely to be processed via their base morphemes, e.g., kirja "book," than derived words formed by more opaque affixes that take two or more allomorphic variants when inflected, e.g., us in kaune-us "beauty," kaune-uden "beauty-genitive-singular." Järvikivi et al. (2006) speculated that the difference could be explained by assuming fast top-down feedback from the lemma to the form level. Essentially the same architecture was recently proposed by Crepaldi et al. (2010). They showed facilitation in masked priming between English irregular inflection like fell-fall (sharing a meaning component), but not with pairs like bell-ball (not sharing a meaning component), a result that contradicts assumptions about strictly blind parsing (see Kelliher and Henderson, 1990). In order to explain this finding they assumed rapid top-down feedback from the lemma to the (orthographic) form level. Even though this model would explain why fell and fall could show effects of interdependency, but semantically unrelated bell and ball would not, it assumes that the architecture for words like corner and farmer is identical. Therefore, it could not account for the (at least numerically) larger masked priming effects for transparent derived than for opaque pseudo complex words, as the framework assumes no lateral communication between the lemma level representations for these words and no feedback from the (morpho)semantic level down (Crepaldi et al., 2010). Importantly, however, the model predicts larger priming effects for transparent inflected words than pseudo complex words, provided that regular inflected (whole word) forms are not assumed to have lemma level representations.

Another possibility to explain graded transparency effects in a hierarchical morphology-first architecture is the hybrid account proposed by Diependaele et al. $(2005,2009)$. The model is an interactive-activation framework that assumes that morphological structure is represented at two levels, sub lexical (morpho)orthographic and supralexical (morpho)semantic, mediated by a lexical form level. When a written word is processed, the visual form is mapped onto the orthographic and the semantic representations in parallel fashion (the latter by the full form representations). A crucial feature of the model is that the different levels are connected via excitatory nodes that can provide both bottom-up and top-down feedback between the levels. An additional feature of the model posits bilateral inhibitory connections at the word form ( lemma) level (Morris et al., 2011). This model allows potential graded effects of morphological priming for opaque words, e.g., brother-broth, to arise via the orthographic level only, whereas priming for more transparent words, like farmer-farm and lion-lions, would benefit from parallel facilitation at the morphosemantic level. Only orthographically related real words, brothel, would suffer from lateral inhibition at the lexical level.

Recent studies seem to support the hybrid account. Diependaele et al. (2009) investigated early morphological processing using prefixed words with mixed intra and cross-modal masked priming with the same procedures as described earlier in order to tap into modality-specific (visual) and modality-free processes. They showed significant visual intramodal priming for prefixed transparent derived Dutch words (Exp. 1), gegil-gil "screamingscream," and pseudo complex words, gebed-bed "prayer-bed," but no facilitation for the form condition, barok-rok "baroque-skirt," but equal significant facilitation for all conditions in cross-modal setting. When the prime-target relation was reversed (Exp. 2), the effect for the pseudo complex condition disappeared with intramodal visual presentation. Further visual-visual experiments showed no effects for any condition with $40 \mathrm{~ms}$ prime duration, but did show significantly larger facilitation for the transparent compared to the opaque condition with $67 \mathrm{~ms}$ prime duration (Exp. 3). Morris et al. (2007) showed larger effects for transparent derived words $(43 \mathrm{~ms})$ than for pseudo complex words $(27 \mathrm{~ms})$ or form controls ( $1 \mathrm{~ms}$ ) in English, showing a significant linear trend with diminishing priming with diminishing transparency. Moreover, they found evidence for both early orthographic segmentation (N250) as well as semantic transparency (N400) in the in the event related potential (ERP) components of the study. Morris et al. (2008) found in a semantic categorization task that 
the early ERP component N250 differentiated orthographic and morphological effects: While the early phase of N250 showed difference between orthographic and transparent/opaque primes, the latter phase of the component showed different electrical activity for opaque and transparent/orthographic primes. Similar activity for transparent and opaque words in the early phase of processing suggests that both of these features are activated and segmented during the short time of prime word presentation: thus, there may be two separate processing phases operating in very close time windows. However, it can also be that the late phase reflects early top-down feedback of semantics, as the authors suggest as well. Interestingly, Morris et al. (2011) showed with visual forward masked priming $(50 \mathrm{~ms})$ that English complex words, flexible, complex non-words, flexity, and orthographically related nonwords, flexire, equally facilitated the recognition of the stem flex. Furthermore, they showed that the effect was the same independent of whether the comparison was with word or a non-word control. The effects were the same on the N250 and N400 components, but in Exp. 2 they found an advantage of morphological complexity (both transparent and opaque) on the N400. It seems that early orthographic segmentation may not be morphologically constrained, but a mere presence of the target in the prime is enough to activate the target word.

\section{CURRENT STUDY}

In order to examine sub- and supralexical effects in complex words, we investigated the relationship between regular transparent inflected words and pseudo complex (opaque) words in morphological processing using visual forward masked $(50 \mathrm{~ms}$ SOA) and auditory-visual cross-modal priming in Finnish; the former seen as tapping the early segmentation phase and the latter the later stage argued to represent the stage of semantic integration (Meunier and Longtin, 2007). In addition, cross-modal priming has been argued to be insensitive to phonological and orthographic overlap between prime and target, measuring instead the extent to which the prime matches the target's amodal lexical representation (e.g., Marslen-Wilson et al., 1994; Boudelaa and Marslen-Wilson, 2005). This allowed us to investigate the relative effects of sub and supra lexical information at the later stage of processing. Moreover, the use of Finnish, with its complex inflectional system and productive derivational and compounding morphology, allowed us to do this in a within-items design, which possibility is not easily available in many of the other frequently studied languages, such as English. We contrasted four conditions in priming a target (see Table 1), such as (1) pseudo complex (monomorphemic) words potentially decomposable into a stem and an existing affix (leijona "lion"), (2) regular inflected words (leijoja "kites"), (3) inflected pseudo words consisting of an illegal combination of a stem and an affix (leijolla), and (4) uninflected pseudo words consisting of a matching stem form followed by a non-affixal phonotactically legal letter string (leijosko). In this set of materials the "stem" is always identical across conditions and will be a bound or a free form $(50 / 50 \%)$ of the target word's morphological paradigm and thus representing a carefully controlled set of stimuli across the conditions.

The predictions for the blind decomposition approach are straightforward (condition numbers refer to Table 1): Significant
Table 1 | Summary of the stimuli in experiments 1 and 2.

\begin{tabular}{llll}
\hline \multicolumn{2}{c}{$\begin{array}{l}\text { Experimental } \\
\text { condition }\end{array}$} & \multicolumn{2}{c}{ Stimuli } \\
\cline { 3 - 4 } & & Prime & Target \\
\hline (1) & Pseudo complex & leijona [Lion-sg-nom] & LEIJA [Kite-sg-nom] \\
(2) & Inflected transparent & leijoja [Kite-pl-ptv] & LEIJA [Kite-sg-nom] \\
(3) & Inflected pseudo & leijolla [Leijo * ade] & LEIJA [Kite-sg-nom] \\
& word & & \\
(4) & Uninflected pseudo & $\begin{array}{l}\text { leijosko [Leijo + non- } \\
\text { suffix] }\end{array}$ & LEIJA [Kite-sg-nom] \\
(5) $\quad$ Word & Unrelated & harput [Harp-pl-nom] & LEIJA [Kite-sg-nom]
\end{tabular}

Sg, singular; pl, plural; nom, nominative case; ptv, partitive case; ade, adessive case; *illegal combination.

facilitation should be obtained for 1-3 but not for 4 in masked priming. Importantly, this facilitation should be equal in magnitude for all experimental conditions, following from the same morpho-orthographic principles across conditions. In crossmodal priming, this account predicts significant facilitation for 2 and 3, but not for 1 and 4 . Note that such illegal combination of a stem and an inflectional affix as in 3 can be said to be strictly interpretable, e.g., a typical inflectional error, and should therefore produce facilitation (Meunier and Longtin, 2007). Furthermore, even though facilitation for these words could be reduced compared to correct inflected words, it should be clearly larger than for words in 1 and 4 , if we take that the effects in cross-modal priming are due to decomposed components and originate at the semantic integration and/or the central level (Marslen-Wilson et al., 1994; Meunier and Longtin, 2007).

However, if we assume that inflected, but not pseudo complex words, benefit from shared stem representation at the lemma level, this predicts greater facilitation for the inflected (2) than the pseudo complex words (1) in masked priming. According to this view, in addition to form-level decomposition, all regularly inflected words are assumed to have a single shared lemma representation, feedback from which would give an additional boost for processing; whereas no lemma node would be shared by pseudo complex words and their (pseudo) stems. Moreover, this view also predicts greater facilitation for the pseudo words in (3) than for (1), because the words in 3 would benefit from morphbased segmentation into stem and affix at the first stage, but do not by definition have separate lemma representations ${ }^{1}$. As above, we expect the transparent inflected words to produce the largest effect. In addition, if it is assumed that form-correspondence can affect cross-modal priming (Allen and Badecker, 2002; BasnightBrown et al., 2007), and that difficulties at integration would cause problems, the somewhat counterintuitive expectation would be

\footnotetext{
${ }^{1}$ We further treated the uninflected pseudo words as a form condition against which we compared the facilitation in other conditions. The results showed that whereas both the real inflected words and the inflected pseudo words differed significantly from the form condition (inflected real: estimate $=-0.057, t=-3.12$, $p \mathrm{MCMC}<0.01$; inflected pseudo: estimate $=-0.049, t=-2.64, p \mathrm{MCMC}<0.01)$, the pseudo complex words did not (estimate $=-0.028, t=-1.49, p=0.137$ ).
} 
more facilitation for (4) than (3); because words in both conditions are identical in terms of form-overlap, but unlike words in (4), words in (3) would suffer from a penalty at morpho-semantic integration. Analogically, least or no facilitation should be observed with (1), which, in addition, would suffer from a penalty at the lemma level already.

The hybrid model predicts facilitation for all conditions at the early phase: the inflected words (2) should show significant facilitation, because both orthographic and semantic information is assumed to become simultaneously activated by the segmented stem and the full form. However, even though it is not entirely clear, whether the model assumes that inflected words too have a lexical level representation. In any case, we can assume that possible lateral inhibition for the inflected words would be minimal or nonexistent, whereas pseudo complex words (1) are predicted to suffer from lateral inhibition at that level, resulting in less facilitation for (1) than (2). Interestingly, since the pseudo words consisting of an illegal combination of a stem and an affix (leijolla) in (3) are formally identical to (1), but do not have lexical representation, it predicts that they result in greater facilitation than the pseudo complex words. As found in Morris et al. (2011), we predict facilitation for words in (4). In turn, cross-modal priming tapping a later stage of processing should show the largest effect for the real inflected words, because they benefit from both the effortless bottom-up morphological segmentation without lexical inhibition and the unambiguous excitatory feedback from lexical and semantic levels. In the light of previous studies, we would expect the rest of the conditions to show greatly diminished facilitation as compared to (2). However, the hybrid model predicts less straightforwardly what would happen in conditions 1,3 , and 4. Interestingly, though, considering bottom-up activation alone, it is possible that greater facilitation would be observed for the inflected pseudo words (3) and pseudo words with an embedded stem form (4), because they would not be subject to lexical level lateral inhibition, unlike the pseudo complex words (1). However, assuming that the model includes a semantic integration mechanism of some sort, then it is possible that the inflected pseudo words might suffer a penalty at this point and pattern with the pseudo complex words.

\section{EXPERIMENT}

In order to examine the sub- and supralexical effects, the present experiment investigated the processing of regular pseudo complex (opaque) words (1) and inflected (transparent) words (2) using visual forward masked priming (50 ms SOA) and audiovisual cross-modal priming. In order to avoid possible effects arising from prime-target form-overlap, we used a withinitems design keeping the stem and form-overlap identical across conditions.

\section{AUTHORIZATION FOR THE USE OF HUMAN SUBJECTS}

The experiments of the present study were non-evasive and were carried out in accordance with Finnish law and adhered to the guidelines of the American Psychological Society and the ethical policies of the University of Helsinki. The present type of (nonmedical) research is exempt under Finnish legislation for ethical review and approval. Participants gave written informed consent to their participation.

\section{MATERIALS AND METHODS Participants}

Altogether 60 undergraduates from the University of Helsinki participated for course credit; 30 in the visual forward masked and 30 in the cross-modal priming experiment. All were native Finnish speakers with normal or corrected-to-normal vision and no reported language deficits.

\section{Materials}

Thirty familiar monomorphemic nouns served as targets in the experiment (mean lemma frequency, 36.5 per million words; length in letters, 4.7). The targets were primed by real and nonwords in five conditions as follows (see Table 1): (1) Pseudo Complex (opaque) words consisting of a real stem (15 free, 15 bound) of the target's inflectional paradigm followed by an affix, derivational, or inflectional (e.g., leijo- is a bound stem form of the lexeme leija "kite"; -na is an essive case suffix). The meaning of the prime was unrelated to the meaning of the target. The correct essive singular form of "kite" would be leijana "as kite" (see Experiment below). (2) Inflected (transparent) - e.g., leijoja-LEIJA ("kites""KITE"): The primes were regular inflected forms of the target. (3) Inflected pseudo words - e.g., leijolla-LEIJA: the primes consisted of a real stem and real inflectional ending in a morphophonologically illegal combination (here, the stem leijo- of the lexeme leija "kite" and -lla, an adessive inflectional suffix; the correct adessive inflection would be leijalla). (4) Uninflected pseudo words - e.g., leijosko-LEIJA: a real inflectional stem followed by a phonotactically legal non-morphological ending (e.g., -sko is not a suffix in Finnish). (5) Unrelated - e.g., harput-LEIJA ("harps"-“KITE"): the primes were inflected nouns phonologically, orthographically, and semantically unrelated to the target.

For the cross-modal priming, the prime words were read aloud by a female speaker in a randomized order and recorded using a high quality condenser microphone (AKG 4000B) and a high quality analog to digital converter (Digidesing Digi002) in an acoustically treated sound studio. The individual words were automatically segmented and each sound file was then manually checked and the segmentation was corrected if needed.

As was shown above, all primes shared the same stem withinitem. In half of the cases the stem was a free form and in half of the cases it was a bound form of the target noun. In the former case the target was completely included in the prime, in the latter case the stem and the target differed with respect to one letter only; either by vowel stem formation (bound stem kaljo- vs. kalja "beer") or consonant gradation (bound stem taki- vs. takki "coat"), both regular morphophonological operations in Finnish. The materials are listed in the Table A1 in Appendix. In addition, 60 real word (monomorphemic nouns) and 90 non-word targets (formed by changing one to three letters from an existing noun) were added, primed by monomorphemic and inflected real words and non-words. In order to diminish the possibility that formoverlap would be informative to the participants, 30 trials had monomorphemic nouns primed by a monomorphemic semantically unrelated minimal pair, e.g., tuli "fire"-TUULI "wind" The 
ratio of the filler trials with a semantically related inflected prime to other prime-target pairs was the same as in the experimental items. There were no further semantically related filler trials.

\section{Design and procedure}

For the masked priming, a fixation point $\left(^{*}\right)$ appeared for $500 \mathrm{~ms}$ followed by a forward mask consisting of a line of hash marks (\#\#\#\#\#\#) presented in the center of the computer screen for $500 \mathrm{~ms}$. Following that, the prime appeared in the same location for $50 \mathrm{~ms}$ in lower case letters, and was followed by the target presented in upper case letters in the same vertical position. The target was presented for $1500 \mathrm{~ms}$ or until the participant made a response, after which started the next sequence with an empty screen for $1500 \mathrm{~ms}$ prior to the fixation point. For the cross-modal priming, a fixation point $(*)$ appeared in the center of the screen for $500 \mathrm{~ms}$ followed by the prime word played through high quality headphones. At the acoustic offset of the prime the target was presented in upper case letters in the center of the screen for $1500 \mathrm{~ms}$ or until the participant made a response, after which the next sequence started with an empty screen for $1500 \mathrm{~ms}$ prior to the fixation point. All stimuli were presented in black 24-point Chicago letters on a light gray background. The participants were instructed to decide as quickly and carefully as possible whether the letter string (following the line of hash marks) was a Finnish word or not by pressing the corresponding "yes" or "no" button on the button box. All participants were tested individually in an experimental room. Twelve practice trials preceded the experiments and an additional 10 filler trials preceded the experimental trials. The prime-target pairs were presented in a randomized order for each participant. Responses and their latencies were recorded for data analyses using E-Prime stimulus presentation software and SRBOX response box.

The items were counterbalanced between five experimental lists so that each list included only one of the above conditions (1-5) per target. All lists included an equal number of trials from each condition. The experimental conditions were therefore within subjects variables and the experiment (visual masked vs. cross-modal) a between subject variable. The participants were assigned to the experimental lists in the order of appearance in both tasks.

\section{RESULTS}

Prior to the data analyses, the following exclusion criteria were used: Targets that resulted in a high number of errors $(>50 \%)$ were discarded: this resulted in three targets to be removed (hippi, kampi, sarka). In addition all incorrect responses in the visual masked and cross-modal experiments (5.6 and 6.9\%, respectively) as well as responses above $1200 \mathrm{~ms}(0.4 \%$ of all data $)$ were removed. The results of the remaining data are summarized in Table 2.

\section{Reaction times}

The remaining RTs were log-transformed and analyzed using linear mixed modeling with participants and items as a crossedrandom factor (e.g., Baayen, 2008), and experiment (visual masked, cross-modal), condition, and stem as fixed-effect predictors. Likelihood ratio tests (ANOVA function in R) showed that by-subject random slopes for the fixed-effect predictors stem and condition did not improve the fit significantly. The best-fitting model is presented in Table 3. The results showed that all conditions induced significant facilitation compared to the unrelated condition. They further showed that words composed of free as opposed to bound stems were responded to faster. However, this effect was in interaction with whether the experiment was masked or cross-modal: Contrasts showed that free stems facilitated recognition compared to bound stems in the former but not in the latter (Cross-modal: estimate $=-0.0717, t=-2.14, p \mathrm{MCMC}=0.0226$; masked: estimate $=-0.0272, t=-0.81, p \mathrm{MCMC}=0.3856$ ). There was a further significant two-way interaction showing that the uninflected pseudo words produced increased facilitation in the crossmodal but not in the masked priming experiment (Cross-modal: estimate $=-0.0710, t=-3.81, p \mathrm{MCMC}=0.0004$; masked: estimate $=-0.0134, t=-0.72, p \mathrm{MCMC}=0.4874)$. Importantly, the results showed no interaction between experiment and the pseudo complex condition. Notably, as can be seen from Table 3, even though the pseudo complex (opaque) words produced significant facilitation when compared to the unrelated condition, the effect was markedly smaller in magnitude than in the inflected real word (transparent) condition. In order to inspect this relation, further analyses were carried out taking the inflected words as the reference level. The results (Table 4) showed that whereas the real inflected words differed significantly from all other conditions, including the pseudo complex, this difference was significantly modulated only by the case of the inflected pseudo words that differed from the inflected real words in the cross-modal but not in the masked priming procedure (Cross-modal: estimate $=0.0619$, $t=3.34, p \mathrm{MCMC}=0.0006$; Masked: estimate $=0.0087, t=0.48$, $p=0.6392$; see text footnote 1 ).

Table 2 | Mean reaction times, error percentages, and net priming effects for masked and cross-modal priming experiments.

\begin{tabular}{|c|c|c|c|c|c|c|}
\hline \multirow[t]{2}{*}{ Prime type } & \multicolumn{3}{|c|}{ Masked priming } & \multicolumn{3}{|c|}{ Cross-modal priming } \\
\hline & RT (Std) & Error $(\%)$ & Effect & RT (Std) & Error $(\%)$ & Effect \\
\hline Pseudo complex & $660(153)$ & 6.5 & -29 & $601(117)$ & 7.7 & -22 \\
\hline Inflected pseudo word & $640(121)$ & 6.0 & -49 & $598(130)$ & 5.9 & -25 \\
\hline Uninflected pseudo word & $675(148)$ & 7.7 & -14 & $583(123)$ & 8.9 & -40 \\
\hline
\end{tabular}

Negative sign denotes facilitation. 
Table 3 | Results from the statistical analyses.

\begin{tabular}{lrlrl}
\hline & Estimate & SE & $\boldsymbol{t}$-Value & $\boldsymbol{p} \mathbf{M C M C}$ \\
\hline (Intercept) & 6.476355 & 0.033943 & 190.80 & $0.0001^{* *}$ \\
Experiment Mask & 0.055084 & 0.035998 & 1.53 & 0.0874 \\
Cond1 & -0.054985 & 0.018563 & -2.96 & $0.0040^{* *}$ \\
Cond2 & -0.118514 & 0.018491 & -6.41 & $0.0001^{*}$ \\
Cond3 & -0.056605 & 0.018466 & -3.07 & $0.0020^{*}$ \\
Cond4 & -0.071027 & 0.018641 & -3.81 & $0.0001^{*}$ \\
StemFree & -0.071709 & 0.033476 & -2.14 & $0.0206^{*}$ \\
Experiment mask:cond 1 & 0.014782 & 0.026320 & 0.56 & 0.5976 \\
Experiment mask:cond 2 & 0.048332 & 0.026103 & 1.85 & 0.0686 \\
Experiment mask:cond 3 & -0.004857 & 0.026171 & -0.19 & 0.8256 \\
Experiment mask:cond 4 & 0.057580 & 0.026404 & 2.18 & $0.0290^{*}$ \\
Experiment mask:stem & 0.044498 & 0.016856 & 2.64 & $0.0096^{*}$ \\
free & & & &
\end{tabular}

Conditions (Cond) are as follows: $1=$ pseudo complex; $2=$ inflected; $3=$ inflected pseudoword; $4=$ uninflected pseudoword. Reference levels are: experiment cross-modal; Condition - unrelated; Stem - bound. ${ }^{*} p<0.05 ;{ }^{*} p<0.001$. Estimates report the regression coefficients for the fixed effects; p-values were obtained by Markov-Chain Monte Carlo (MCMC) sampling with 10000 replications.

Table 4 | Results from statistical analyses with inflected words as reference level.

\begin{tabular}{lrlrl}
\hline & Estimate & SE & $\boldsymbol{t}$-Value & $\boldsymbol{p M C M C}$ \\
\hline (Intercept) & 6.357841 & 0.033928 & 187.39 & $0.0001^{* *}$ \\
Experiment mask & 0.103417 & 0.035868 & 2.88 & $0.0012^{*}$ \\
Cond1 & 0.063529 & 0.018583 & 3.42 & $0.0006^{* *}$ \\
Cond3 & 0.061909 & 0.018523 & 3.34 & $0.0004^{* *}$ \\
Cond4 & 0.047487 & 0.018676 & 2.54 & $0.0102^{*}$ \\
Cond5 & 0.118514 & 0.018491 & 6.41 & $0.0001^{*}$ \\
Stem Free & -0.071709 & 0.033476 & -2.14 & $0.0210^{*}$ \\
Experiment mask:cond 1 & -0.033550 & 0.026181 & -1.28 & 0.1904 \\
Experiment mask:cond 3 & -0.053190 & 0.026072 & -2.04 & $0.0450^{*}$ \\
Experiment mask:cond 4 & 0.009248 & 0.026262 & 0.35 & 0.7402 \\
Experiment mask:cond 5 & -0.048332 & 0.026103 & -1.85 & 0.0642 \\
Experiment mask:stem & 0.044498 & 0.016856 & 2.64 & $0.0088^{*}$ \\
free & & & & \\
\hline
\end{tabular}

Conditions (Cond) are as follows: $1=$ pseudo complex; $3=$ inflected pseudoword; $4=$ uninflected pseudoword; $5=$ unrelated. Reference levels are: experiment-cross-modal; Condition - inflected; Stem - bound. ${ }^{*} p<0.05$; ${ }^{*} p<0.001$. Estimates report the regression coefficients for the fixed effects; $p$-values were obtained by Markov-Chain Monte Carlo (MCMC) sampling with 10000 replications.

\section{Error rates}

We inspected the error data by fitting a logistic mixed effects regression model (lmer in $\mathrm{R}$ with binomial family) with subjects and items as a crossed-random factor (e.g., Baayen, 2008; Jaeger, 2008), with experiment, condition, and stem type (bound, free) as fixed-effect predictors. The model revealed no differences between conditions $(z s<1.05)$ and no effect of stem type $(z s<1)$. However, the inflected condition produced less errors in the masked procedure than the others conditions (inflected: estimate $=-1.117, z=-1.889, p=0.058$; other conditions, all ps $>0.24)$.

\section{GENERAL DISCUSSION}

Overall the results fit the graded pattern observed in earlier studies showing diminishing facilitation with decreasing morphological transparency in early processing. Regular inflected words (leijoja "kites") showed the largest facilitation in both masked and cross-modal priming. Importantly, however, in masked priming pseudo words consisting of an illegal combination of a stem and an affix (leijolla) facilitated processing as much as regular inflected words and more than pseudo complex words. Furthermore, even though pseudo complex words showed significant priming, the effect was weaker than for inflected pseudo words. This is important, since formally illegally combined pseudo words such as leijolla are exactly like pseudo complex words (leijona "lion"), namely illegal combinations of a stem and an affix. There is but one crucial exception; unlike the former words, pseudo complex words have a whole word meaning and thus an existing lexical representation. Importantly, unlike with regular inflected words, this meaning is not related to the meaning of the (decomposed) stem. Given the blind decomposition framework, there is no principled reason why pseudo complex words should be processed differently from the other two. At a later stage, both words patterned together, showing significant but diminished priming compared to inflected words. The orthographic pseudo words, consisting of a related stem form and a non-suffixal ending, showed the smallest effect in masked priming, but, interestingly, large $40 \mathrm{~ms}$ facilitation in the cross-modal experiment. Thus not only did we find significant facilitation in the absence of strict interpretability, but also significant facilitation for non-words composed of a real stem and non-suffix ending. This result seems potentially incompatible with the classical assumption that cross-modal priming is largely insensitive to mere form-based effects. In contrast, it seems that salient enough bottom-up form information can be enough to facilitate processing even in cross-modal priming, a conclusion argued recently by Allen and Badecker (2002) and Basnight-Brown et al. (2007). Importantly, it seems that semantic/syntactic compatibility of the component morphemes is not a precursor for significant facilitation to arise (cf. Meunier and Longtin, 2007).

Therefore, facilitation in the present study was clearly modulated both by whether the primes could be segmented into existing morph(eme)s and whether they had surface meanings that were different from the meaning of their decomposed (pseudo) stems, in which case processing was impeded. The results thus confirm in a within-item design the earlier observation that even though morphologically opaque pseudo complex words induce significant priming, the effect is smaller than with transparent complex words, and markedly so, if the comparison is with inflected rather than derived forms. The current findings are clearly incompatible with strict morphology-second approaches that assume that all morphological effects arise only after word level processing is completed. The recent debate between morphology-first and morphology-second approaches has been greatly influenced by the robust finding that pseudo complex words like corner (corn) induce significant priming under masked conditions, but words 
like turnip (turn) do not, pointing to a need to posit an early sublexical morph(eme)-based segmentation phase that operates on these morphologically motivated sublexical units. However, our results are also incompatible with this blind feed-forward decomposition account, because it would assume no difference between regular inflected and pseudo complex words or between these and inflected pseudo words and could not account for our results or the graded effects quite convincingly shown also in the previous literature. Such effects are, however, explicitly predicted and reconcilable in multi-level frameworks that represent morphological information at separate interactive form and lemma/lexical levels, thus explaining the result as originating from the lemma rather than the semantic level.

The hybrid model could in principle allow for the mere formbased effect to arise as bottom-up facilitation, because such letter strings would proceed unhindered by any level from the letter level up (Morris et al., 2011). However, they would also not benefit from any facilitative feedback, which would explain the diminished effect with respect to regular words. Moreover, the hybrid account (Diependaele et al., 2005, 2009) also allows the graded effects of morphological priming that correlate with graded opacity, turnipcorner-farmer-lions, here to arise via lexical (or lemma) level lateral inhibition. However, this is provided that for regular inflections the lateral inhibition is practically nil (or, as is assumed in many lemma-based models, that regular inflected words tend not to have separate full form representations). Also, because the model allows semantic information to feed back down, it may be that regular inflections would benefit from this top-down facilitation as well. This seems a fitting account at least for our cross-modal results. Note, that this model does not assume that sublexical form and supralexical semantic information need to influence processing at the same time. It is thus compatible with an early orthographic segmentation phase, even though it does suggest that supralexical semantic information can be detectable at a very early stage, even with masked priming. We will turn to this assumption next.

\section{EARLY INFLUENCE OF MEANING?}

Even though the present results from masked priming are in line with the early form-based segmentation view, they are not explainable from morpho-orthography alone, because semantic unrelatedness of the prime's whole word reading seemed to have a consistent negative influence. In order to assess this possibility, we inspected the response data of the pseudo complex words in masked priming separately by inspecting the influence of various frequency-based measures on the priming results. We were particularly interested in morphological family size, the type frequency with which a word occurs as a constituent in derived and compound words, because it is known to reflect post-lexical-semantic activation spreading in the network of family members in several languages, including Finnish (e.g., Schreuder and Baayen, 1997; Bertram et al., 2000; De Jong et al., 2000; Moscoso del Prado Martín et al., 2004, 2005; but see Davis and Rastle, 2010).

As above, we inspected the log-transformed reaction time data to pseudo complex words using a linear mixed-effect model with subjects and items as a crossed-random factor and log target frequency, log target family size, log prime frequency, as well as log prime family size as fixed-effect predictors. We removed two target words that resulted in a high number of errors $(>50 \%$ for hippi and sarka) in the pseudo complex condition. Because some of the pseudo complex primes had derivational suffixes, we also used suffix type (derivational, inflectional) as an additional predictor. The results showed that suffix type (inflected, derived) had no effect $(t=-0.40)$ and was dropped. The resulting model revealed both a facilitative effect of $\log$ prime frequency $(t=-2.41$, $p \mathrm{MCMC}=0.017)$ as well as an inhibitory effect of log prime family size $(t=2.09, p \mathrm{MCMC}=0.035)$. Both target frequency and target family size had a marginal facilitative effect $(t=-1.68$; $t=-1.94, p s<0.1$, respectively). This indicates that whereas the formal properties of (opaque) pseudo complex words facilitate the processing of the target, the inhibitory family size effect suggests that the sublexical properties of the pseudo complex words interfere with the processing of the target stem. However, as is often the case with family size and (lemma) frequency (e.g., De Jong et al., 2000; Baayen, 2008), these two frequency measures had a fairly high (negative) correlation in our data (coefficient $=-0.798$ ). In order to inspect the relative impact of prime family size with respect to prime frequency, we created a new predictor VF2Fratio by subtracting the log lemma frequency from the log family size for the prime words. Further analyses with the new predictor showed that the ratio between prime family size and prime frequency was a significant predictor of the reaction time data to pseudo complex words $(t=2.46, p \mathrm{MCMC}=0.017)$ while the target-based frequency measures remained marginal ( $p s>0.05$ ). Furthermore, the new model also resulted in a significantly better fit to the data than the previous one (likelihood ratio test, $p=0.000014)$. This result indicates that not only does prime family size inhibit the processing of the target words, but also its relative effect increases when the relative size of the word's morphological family as compared to the word's frequency increases.

The importance of semantic information at the early stage of processing has been recently argued by Feldman and colleagues in several studies (e.g., Feldman and Basnight-Brown, 2008; Feldman et al., 2009; Pastizzo and Feldman, 2009; but see Davis and Rastle, 2010, for a critique). Even though robust lexical-semantic effects are hard to show in masked priming, they are not completely non-existent (see Van den Bussche et al., 2009 for an overview), and there is evidence that semantic transparency affects masked priming results at least in some languages (e.g., Boudelaa and Marslen-Wilson, 2005 for evidence from Arabic). In principle then, semantic information can affect the early stage of processing. This raises the following questions: is this reason enough to abandon the form-first view, what would be the locus of the semantic effect, and what does semantics mean in this context?

One way to approach the questions is to assume that morphological information is represented at several stages, corresponding to form information (orthography) and higher-level, whether semantic or lexical, that mediates between form and the semantic system, therefore making - at least certain types of - semantic effects possible already at the early stages of decomposition. Moreover, recent studies have assumed top-down feedback between two consecutive levels of representation in this type of architecture (Järvikivi et al., 2006; Crepaldi et al., 2010). In Crepaldi et al. (2010), priming can originate both from the decomposed formlevel representations and from the lemma level. The latter level 
would store lexical entries as defined by their meaning and certain syntactic properties, like grammatical class. Most inflected forms would thus share a single lemma node at this level, which would not be the case with derived forms (see also e.g., Aronoff, 1976, 1994; Anderson, 1992, for lexeme-based theories of morphology). However, for pseudo complex words, like leijona "lion," that are in fact monomorphemic, the lemma level would be shared among all their inflected forms but would have no connection to the pseudo stem, leija "kite," that shares no meaning with the former. This model would then predict that pseudo complex words would show greater priming than words with a real stem but a non-affixal ending, like brothel, but would be largely indistinguishable from true derived forms like bomber. In addition, the model would explain why both ambiguous inflected forms (aitoja "fences" or "genuines") as well as inflected forms that are unambiguous (aito-na "as-genuine") but share a stem form with the target's morphological paradigm (aita "a fence") induce comparable priming to that observed with unambiguous inflected forms (aitana "as fence"; Järvikivi et al., 2009; see also Badecker and Allen, 2002). Furthermore, it would also predict the significant facilitation relative to form controls observed for free standing bound stem allomorphs in masked and unmasked visual priming (Järvikivi and Niemi, 2002, 2003). Most importantly, however, the model explicitly predicts that regular inflected forms should induce stronger facilitation than pseudo complex words. This finding was first demonstrated in the present study.

Importantly, however, the two-level model cannot capture the observed inhibitory family size influence induced by the pseudo complex primes - unless it allows this effect to originate from the semantic level. It cannot be caused by lemma level feedback, because the model explicitly assumes no connections between derived (and probably compound) forms at this stage. If we assume that the effect of family size is semantic in nature, as argued by a host of studies (Baayen et al., 2006 and the references therein), then it seems that semantic influences appear rapidly and affect morphological processing very early. This speaks of a specifically morpho-semantic top-down influence that could affect processing very quickly.

\section{INTERPRETING FAMILY SIZE EFFECTS}

Our results would fit well with the view that supralexical information plays a role from early on: This would explain why a mismatching whole word reading would diminish the facilitation with respect to regular inflection. Importantly, our regression analysis further suggested that morphological family size of the pseudo complex primes had a role to play via an inhibitory effect, whereas its lemma frequency facilitated processing. Because lemma and surface frequencies are highly correlated, the latter effect is most naturally interpreted as indicative of early form-based facilitation. However, even though family size effects have been demonstrated before in masked priming (e.g., McCormick et al., 2009 for an effect of stem family size), this is the first time that prime family size has been shown to have an inhibitory effect in masked priming, possibly enhanced by the relatively large families in Finnish (Moscoso del Prado Martín et al., 2004) compared to languages like English. However, it is not yet clear whether the current evidence is in favor of abandoning the early morpho-orthographic morph-based view of decomposition or whether it is simply showing that masked priming is sensitive to semantic processes that might kick off earlier than thought before and might even originate from activation feedback from higher levels (Davis and Rastle, 2010). Therefore, we first have to establish whether such effects as were reported in this study and in previous ones could be reconciled in morphology-first models.

As a first approximation, the hierarchical interactive-activation account (Diependaele et al., 2009; Morris et al., 2011) offers a possibility to interpret the family size inhibition for the pseudo complex words. Assuming that rapid supralexical feedback from the morpho-semantic level boosts the lexical level representations for the (pseudo) complex word and its stem, it follows that the lateral inhibition mechanism would favor the pseudo complex words over its stem representation more than in the case of transparent derived words that are related in meaning to their stems. In other words, feedback from the semantic level would boost the whole word reading of the pseudo complex words at the lexical level and the increased competition would be reflected as diminished priming. Importantly, as regular inflections would benefit from maximal top-down feedback with minimal or no lateral inhibition, the processing advantage for these words follows from this framework as well. The account also predicts an advantage for productive derived words over pseudo complex words, other things being equal, which is indeed suggested by many previous studies (see Feldman et al., 2009, for an overview). In short, the hybrid model as sketched here, incorporates an early orthographic segmentation phase, but implies that supralexical semantic information can be detectable from a very early on and can affect masked priming results, without assuming that this influence is exerted immediately at the exposure to the word form. Rather, the locus of the supralexical effect would be at the lexical level and would reflect the pseudo complex word's conceptual relationship with its morphological family members.

Many people agree that in order to explain the processing of complex words, and pseudo complex words in particular, an appropriate model, like the one above, needs to assume a morphologically motivated segmentation component. Against this background, it is interesting to ask how far a simple feed-forward model that assumes no morphological representations at any level would fair. A recent paper by Baayen et al. (2011) attempts to answer this by investigating morphological processing in a computational model, a naïve discriminative learner. The model is a probabilistic feed-forward architecture that assumes a direct form-tomeaning mapping with symbolic representation for orthographic and semantic information, but assumes no morpheme-based or whole word representations and no mediating stage between the two levels. At the form level the representations consist of single letters and letter bigrams. At the semantic level the authors assume meaning representations for words, derivational affixes and various morphosyntactic features, such as case and number. As a more detailed presentation of the model is beyond the scope of the present paper, the reader is referred to the original work. However, it is noteworthy that the authors are able to model a host of established results in the literature, such as frequency, family size, and paradigm effects without assuming higher-level structured representations. Interestingly, they also modeled the masked priming 
study of Rastle et al. (2004) and showed highly significant priming effects for derived and pseudo derived words, which, importantly, were statistically indistinguishable from each other as in the original study. How form controls, like turnip, would fare in this procedure is left untold. It seems then that the pseudo derivation effect can be reproduced in the absence of pre-lexical segmentation.

As the recent reviews (Rastle and Davis, 2008; Feldman et al., 2009) arrive at opposite conclusions, it may be too early to claim a stake on when exactly supralexical information affects morphological processing, or whether semantic effects arise at the same time with formal ones or not. Furthermore, as shown by Baayen et al. (2011), it may yet again be too early to close the case on morphological parsing. The effects that look like originating from morphological representations and can conveniently be referred to as such using discrete labels, need not and may originate from such in cognitive reality. Even though these issues are for further research to resolve, the present findings suggest that inflected words are processed with more ease than pseudo complex words and masked priming is influenced by the morphological family size of the (pseudo complex) prime the most likely interpretation of which effect would be semantic.

\section{CONCLUSION}

In general, the present study is in line with the assumption that sublexical morph(eme)-like units are activated by an early rapid

\section{REFERENCES}

Allen, M., and Badecker, W. (1999). Stem homograph inhibition and stem allomorphy: representing and processing inflected forms in a multilevel lexical system. J. Mem. Lang. $41,105-123$.

Allen, M., and Badecker, W. (2002). Inflectional regularity: probing the nature of lexical representation in a cross-modal priming task. J. Mem. Lang. 46, 705-722.

Anderson, S. (1992). A-Morphous Morphology. Cambridge: CUP.

Aronoff, M. (1976). Word Formation in Generative Grammar. Cambridge, MA: MIT Press.

Aronoff, M. (1994). Morphology by Itself: Stems and Inflectional Classes. Cambridge, MA: MIT Press.

Baayen, H., Dijkstra, T., and Schreuder, R. (1997). Singulars and plurals in Dutch: evidence for a parallel dualroute model. J. Mem. Lang. 37, 94-117.

Baayen, R. H. (2008). Analyzing Linguistic Data: A Practical Introduction to Statistics Using R. Cambridge, UK: Cambridge University Press.

Baayen, R. H., Feldman, L. F., and Schreuder, R. (2006). Morphological influences on the recognition of monosyllabic monomorphemic words. J. Mem. Lang. 53, 496-512.

Baayen, R. H., Milin, P., Đurđević, D. F., Hendrix, P., and Marelli, M. (2011). An amorphous model for morphological processing in visual comprehension based on naïve discriminative learning. Psychol. Rev. 118, 438-481.

Badecker, W., and Allen, M. (2002). Morphological parsing and the perception of lexical identity: a masked priming study of stem homographs. J. Mem. Lang. 47, 125-144.

Basnight-Brown, D. M., Chen, L., Hua, S., Kostic, A., and Feldman, L. B. (2007). Monolingual and bilingual recognition of regular and irregular English verbs: sensitivity to form similarity varies with first language experience. J. Mem. Lang. 57, 65-80.

Bertram, R., Baayen, R. H., and Schreuder, R. (2000). Effects of family size for complex words. J. Mem. Lang. 42, 390-405.

Bodner, G. E., and Masson, M. E. J. (2003). Beyond spreading activation: an influence of relatedness proportion on masked semantic priming. Psychon. Bull. Rev. 10, 645-652.

Boudelaa, S., and Marslen-Wilson, W. D. (2005). Discontinuous morphology in time: incremental masked priming in Arabic. Lang. Cogn. process. 20, 207-260.

Bowers, J. S., Davis, C. J., and Hanley, D. A. (2005). Automatic semantic activation of embedded words: is there a "hat" in "that"? J. Mem. Lang. 52, 131-143.

Crepaldi, D., Rastle, K., Coltheart, M., and Nickels, L. (2010). 'Fell' primes

segmentation procedure operating on those units. However, the present study showed that the early stage of morphological processing is not only sensitive to whether the orthographic string can be segmented into an existing stem and affix, but also whether the full form is an existing word the meaning of which differs from the meaning of the segmented stem. It is thus likely that from early on morphological processing is not governed by morpho-orthographic processes alone, but is most likely sensitive to higher order top-down information, perhaps originating from the supralexical semantic level representations. As the lemma level or lexical level representations are seen to be semantically motivated lexical entries, activation at this level would then be the locus of the observed whole word meaning based penalty for pseudo complex compared to inflected words. This type of architecture would explain the primacy of regular inflection at this stage. Moreover, the observed influence of morphological family size on the processing of pseudo complex words suggests that even at this stage higher semantic processes are going on. Whether semantic information kicks off at the same time or slightly later than form information is a further question to investigate.

\section{ACKNOWLEDGMENTS}

We would like to thank Martti Vainio and Leena Wahlberg for her help with data collection and Harald Baayen for his suggestions on data analyses.

'fall', but does 'bell' prime 'ball'? Masked priming with irregularlyinflected primes. J. Mem. Lang. 63 , 83-99.

Davis, M. H., and Rastle, K. (2010). Form and meaning in early morphological processing: comment on Feldman, O'Connor, and Moscoso del Prado Martin. Psychon. Bull. Rev. 17, 749-755.

De Jong, N. H., Schreuder, R., and Baayen, R. H. (2000). The morphological family size effect and morphology. Lang. Cogn. Process. 15, 329-365.

Diependaele, K., Sandra, D., and Grainger, J. (2005). Masked crossmodal morphological priming: unravelling morpho-orthographic and morpho- semantic influences in early word recognition. Lang. Cogn. Process. 20, 75-114.

Diependaele, K., Sandra, D., and Grainger, J. (2009). Semantic transparency and masked morphological priming: the case of prefixed words. Mem. Cognit. 37, 895-908.

Feldman, L. B., and Basnight-Brown, D. M. (2008). List context fosters semantic processing: parallels between semantic and morphological facilitation when primes are forward masked. J. Exp. Psychol. Learn. Mem. Cogn. 34, 680-687.

Feldman, L. B., O'Connor, P. A., and Moscoso del Prado Martín, F.
(2009). Early morphological processing is morpho-semantic and not simply morpho-orthographic: an exception to form-then-meaning accounts of word recognition. Psychon. Bull. Rev. 16, 684-691.

Giraudo, H., and Grainger, J. (2000). Effects of prime word frequency and cumulative root frequency in masked morphological priming. Lang. Cogn. Process. 15, 421-444.

Günther, H. (1988). Oblique word forms in visual word recognition. Linguistics 26, 583-600.

Jackendoff, R. (1975). Semantic and morphological regularities in the lexicon. Language 51, 639-671.

Jaeger, T. F. (2008). Categorical data analysis: away from ANOVAs (transformations or not) and towards logit mixed models. J. Mem. Lang. 59, 434-446.

Järvikivi, J., Bertram, R., and Niemi, J. (2006). Affixal salience and the processing of derivational morphology: the role of suffix allomorphy. Lang. Cogn. Process. 21, 394-431.

Järvikivi, J., and Niemi, J. (2002). Formbased representation in the mental lexicon: priming (with) bound stem allomorphs in Finnish. Brain Lang. 81, 412-423.

Järvikivi, J., and Niemi, J. (2003). Allomorphs as paradigm indices: on-line experiments with Finnish free and bound stems. SKY J. Linguist. 15, 119-143. 
Järvikivi, J., Pyykkönen, P., and Niemi, J. (2009). Exploiting degrees of inflectional ambiguity: stem form and the time course of morphological processing. J. Exp. Psychol. Learn. Mem. Cogn. 35, 221-237.

Kelliher, S., and Henderson, L. (1990). Morphology based frequency effects in the recognition of irregularly inflected words. Br. J. Psychol. 81, 527-539.

Levelt, W. J. M., Roelofs, A., and Meyer, A. S. (1999). A theory of lexical access in speech production. Behav. Brain Sci. 22, 1-75.

Longtin, C.-M., and Meunier, F. (2005). Morphological decomposition in early visual word processing. J. Mem. Lang. 53, 26-41.

Longtin, C. M., Segui, J., and Halle, P. (2003). Morphological priming without morphological relationship. Lang. Cogn. Process. 18, 313-334.

Marslen-Wilson, W. D., Bozic, M., and Randall, B. (2008). Early decomposition in visual word recognition: dissociating morphology, form, and meaning. Lang. Cogn. Process. 23, 394-421.

Marslen-Wilson, W. D., Tyler, L. K., Waksler, R., and Older, L. (1994). Morphology and meaning in the English mental lexicon. Psychol. Rev. 101, 3-33.

McCormick, S. F., Rastle, K., and Davis, M. H. (2009). Adore-able not adorable? Orthographic underspecification studied with masked repetition priming. Eur. J. Cogn. Psychol. 21, 813-836.
Meunier, F., and Longtin, C.-M. (2007). Morphological decomposition and semantic integration in word processing. J. Mem. Lang. 56, 457-471.

Morris, J., Frank, T., Grainger, J., and Holcomb, P. J. (2007). Semantic transparency and masked morphological priming: an ERP investigation. Psychophysiology 44, 506-521.

Morris, J., Holcomb, P. J., and Grainger, J. (2008). An electrophysiological investigation of early effects of masked morphological priming. Lang. Cogn. Process. 23, 1021-1056.

Morris, J., Porter, J. H., Grainger, J., and Holcomb, P. J. (2011). Effects of lexical status and morphological complexity in masked priming: an ERP study. Lang. Cogn. Process. 26, 558-599.

Moscoso del Prado Martín, F., Bertram, R., Häikiö, T., Schreuder, R., and Baayen, R. H. (2004). Morphological family size in a morphologically rich language: the case of Finnish compared with Dutch and Hebrew. J. Exp. Psychol. Learn. Mem. Cogn. 30, 1271-1278.

Moscoso del Prado Martín, F., Deutsch, A., Frost, R., Schreuder, R., De Jong, N. H., and Baayen, R. H. (2005). Changing places: a cross-language perspective on frequency and family size in Dutch and Hebrew. J. Mem. Lang. 53, 496-512.

Pastizzo, M. J., and Feldman, L. B. (2009). Boats prime floats but coats don't: priming from nonmorphological form and meaning similarity invites a computational account of morphological processing. Ment. Lex. 4, 1-25.

Perea, M., and Gotor, A. (1997). Associative and semantic priming effects occur at very short stimulus-onset asynchronies in lexical decision and naming. Cognition 62, 223-240.

Plaut, D. C., and Gonnerman, L. M. (2000). Are non-semantic morphological effects incompatible with a distributed connectionist approach to lexical processing. Lang. Cogn Process. 15, 445-485.

Rastle, K., and Davis, M. H. (2008). Morphological decomposition based on the analysis of orthography. Lang. Cogn. Process. 23, 942-971.

Rastle, K., Davis, M. H., and New, B. (2004). The broth is my brothel's brother: morpho-orthographic segmentation in visual word recognition. Psychon. Bull. Rev. 11, 1090-1098.

Schreuder, R., and Baayen, R. H. (1995). "Modeling morphological processing," in Morphological Aspects of Language Processing, ed. L. B. Feldman (Hillsdale, NJ: Erlbaum), 131-154.

Schreuder, R., and Baayen, R. H. (1997). How complex simplex words can be. J. Mem. Lang. 36, 118-139.

Solomyak, O., and Marantz, A. (2009). Lexical access in early stages of visual word processing: a singletrial correlational MEG study of heteronym recognition. Brain Lang. 108, 191-196.

Taft, M. (1991). Reading and The Mental Lexicon. Hove: Lawrence Erlbaum.
Taft, M., and Kougious, P. (2004). The processing of morpheme-like units in monomorphemic words. Brain Lang. 90, 9-16.

Taft, M., and Nguyen-Hoan, M. (2010). A sticky stick? The locus of morphological representation in the lexicon. Lang. Cogn. Process. 25, 277-296.

Van den Bussche, E., Van den Noortgate, W., and Reynvoet, B. (2009). Mechanisms of masked priming: a meta-analysis. Psychol. Bull. 135, 452-477.

Conflict of Interest Statement: The authors declare that the research was conducted in the absence of any commercial or financial relationships that could be construed as a potential conflict of interest.

Received: 20 May 2011; accepted: 07 October 2011; published online: 25 October 2011.

Citation: Järvikivi J and Pyykkönen $P$ (2011) Sub- and supralexical information in early phases of lexical access. Front. Psychology 2:282. doi: 10.3389/fpsyg.2011.00282

This article was submitted to Frontiers in Language Sciences, a specialty of Frontiers in Psychology.

Copyright (c) 2011 Järvikivi and Pyykkönen. This is an open-access article subject to a non-exclusive license between the authors and Frontiers Media SA, which permits use, distribution and reproduction in other forums, provided the original authors and source are credited and other Frontiers conditions are complied with. 


\section{APPENDIX}

Table A1 | Stimuli used in the present experiments.

\begin{tabular}{|c|c|c|c|c|c|c|c|c|c|}
\hline \multicolumn{2}{|c|}{ Pseudo complex } & \multicolumn{2}{|l|}{ Inflected } & \multirow{2}{*}{$\begin{array}{l}\text { Non-word } 1 \\
\text { Akajen }\end{array}$} & \multirow{2}{*}{$\begin{array}{l}\text { Non-word } 2 \\
\text { Akavos }\end{array}$} & \multicolumn{2}{|c|}{ Unrelated } & \multicolumn{2}{|l|}{ Target } \\
\hline Akana & "Husk" & Akalle & "Old-woman-all-sg" & & & Singot & “Bazooka-nom-pl” & Akka & "Old woman" \\
\hline Hipiä & "Skin" & Hipit & “Hippie-nom-pl” & Hipinä & Hipie & Hiipat & "Mitre-nom-pl" & Hippi & “Hippie" \\
\hline Jalava & "Elm" & Jalassa & "Foot-ine-sg" & Jalana & Jalape & Kurssina & "Course-ess-sg" & Jalka & "Foot" \\
\hline Kaljama & "Slippery ice" & Kaljana & "Beer-ess-sg" & Kaljaja & Kaljano & Ruohona & "Grass-ess-sg" & Kalja & "Beer" \\
\hline Kampela & "Flounder" & Kampea & "Crank-ptv-sg" & Kampella & Kampemmi & Läpät & “Valve-nom-pl" & Kampi & "Crank" \\
\hline Kanava & "Canal" & Kanana & "Chicken-ess-sg" & Kanajen & Kanappi & Tasetta & "Balance-ptv-sg" & Kana & "Chicken" \\
\hline Kärsämö & "Yarrow" & Kärsänä & "Trunk-ess-sg" & Kärsäen & Kärsäli & Pestille & "Job-all-sg" & Kärsä & "Trunk" \\
\hline Kattila & "Saucepan" & Kattina & "Cat-ess-sg" & Kattilla & Kattitsi & Uuneja & "Oven-ptv-pl" & Katti & "Cat" \\
\hline Kauhava & "Place" & Kauhana & "Scoop-ess-sg" & Kauhajen & Kauhalo & Lankona & "Brother-in-law-ess-sg" & Kauha & "Scoop" \\
\hline Korona & "Corona" & Korot & "Heel-nom-pl" & Koroja & Korono & Rannat & "Shore-nom-pl" & Korko & "Heel" \\
\hline Lakana & "Sheet" & Lakalla & “Lacquer-ade-sg" & Lakajen & Lakara & Pannuun & "Pan-ill-sg" & Lakka & "Lacquer" \\
\hline Leijona & "Lion" & Leijoja & "Kite-ptv-pl" & Leijolla & Leijosko & Harput & "Harp-nom-pl" & Leija & "Kite" \\
\hline Lomake & "Form" & Lomalla & "Holiday-ade-sg" & Lomaita & Lomarki & Kaavana & "Formula-ess-sg" & Loma & "Holiday" \\
\hline Majava & "Beaver" & Majana & "Hut-ess-sg" & Majajen & Majaro & Lemuja & "Smell-ptv-pl" & Maja & "Hut" \\
\hline Mammona & "Riches" & Mammoja & "Mother-ptv-pl" & Mammolle & Mammokar & Kasinot & "Casino-nom-pl" & Mamma & "Mother" \\
\hline Meloni & "Melon" & Meloja & "Paddle-ptv-pl" & Melolla & Melote & Eliönä & "Organism-ess-sg" & Mela & "Paddle" \\
\hline Moukari & "Sledgehammer" & Moukan & “Lout-gen-sg" & Moukana & Moukaro & Elukat & "Animal-nom-pl" & Moukka & "Lout" \\
\hline Pakina & "Causerie" & Pakissa & "Toolbox-ine-sg" & Pakien & Pakino & Sudesta & "Wolf-ela-sg" & Pakki & "Reverse" \\
\hline Papana & "Dropping" & Papalle & "Grandfather-all-sg" & Papaista & Paparo & Konttiin & "Container-ill-sg" & Pappa & "Grandfather" \\
\hline Patina & "Verdigris" & Patit & "Bump-nom-pl" & Patija & Patinu & Harat & "Harrow-nom-pl" & Patti & "Bump" \\
\hline Pernaja & "Place" & Pernana & "Spleen-ess-sg" & Pernajen & Pernatsi & Hirsiä & "Log-ptv-pl" & Perna & "Spleen" \\
\hline Pilari & "Pillar" & Pilat & "Prank-nom-sg" & Pilaja & Pilano & Heimot & "Tribe-nom-pl" & Pila & "Prank" \\
\hline Pokkari & "Paperback" & Pokkana & "Poker (face)-ess-sg" & Pokkan & Pokkalo & Vehnänä & "Wheat-ess-sg" & Pokka & "Poker (face)" \\
\hline Puntari & "Scale" & Puntaa & "Pound-ptv-sg" & Puntan & Puntaro & Hylkyä & "Wreck-ptv-sg" & Punta & "Pound" \\
\hline Sarana & "Hinge" & Saralla & "Strip-ade-sg" & Sarailla & Sarakso & Frangit & "Frank-nom-pl" & Sarka & "Strip" \\
\hline Satula & "Saddle" & Satuja & "Fairytale-ptv-pl" & Satuita & Satuski & Junana & "Train-ess-sg" & Satu & "Fairytale" \\
\hline Sikari & "Cigar" & Sikana & "Pig-ess-sg" & Sikan & Sikapo & Hiekat & "Sand-nom-pl" & Sika & "Pig" \\
\hline Takila & "Rog" & Takitta & "Coat-abe-sg" & Takiin & Takito & Mafiaa & "Mafia-pt-sg" & Takki & "Coat" \\
\hline Vanaja & "Place" & Vanana & "Trail-ess-sg" & Vanajen & Vanappi & Kilit & "Goat-nom-pl" & Vana & "Trail" \\
\hline Vasama & "Bolt" & Vasana & "Calf-ess-sg" & Vasaja & Vasate & Pajut & "Willow-nom-pl" & Vasa & "Calf" \\
\hline
\end{tabular}

Nom, nominative; gen, genitive; ptv, partitive; ess, essive; ine, inessive; ade, adessive; all, allative; abe, abessive; ill, illative; sg, singular; pl, plural. 